\title{
Self-Gated Fat-Suppressed Cardiac Cine MRI
}

\author{
R. Reeve Ingle ${ }^{1}$, Juan M. Santos ${ }^{2}$, William R. Overall ${ }^{2}$, Michael V. McConnell ${ }^{1,3}$, Bob S. \\ $\mathrm{Hu}^{1,2,4}$, and Dwight G. Nishimura ${ }^{1}$ \\ ${ }^{1}$ Magnetic Resonance Systems Research Laboratory, Department of Electrical Engineering, \\ Stanford University, Stanford, California, USA \\ ${ }^{2}$ HeartVista, Inc., Menlo Park, California, USA \\ ${ }^{3}$ Division of Cardiovascular Medicine, Stanford University, Stanford, California, USA \\ ${ }^{4}$ Palo Alto Medical Foundation, Palo Alto, California, USA
}

\section{Abstract}

Purpose-To develop a self-gated alternating repetition time balanced steady-state free precession (ATR-SSFP) pulse sequence for fat-suppressed cardiac cine imaging.

Methods-Cardiac gating is computed retrospectively using acquired MR self-gating data, enabling cine imaging without the need for electrocardiogram (ECG) gating. Modification of the slice-select rephasing gradients of an ATR-SSFP sequence enables the acquisition of a 1D selfgating readout during the unused short repetition time (TR). Self-gating readouts are acquired during every TR of segmented, breath-held cardiac scans. A template-matching algorithm is designed to compute cardiac trigger points from the self-gating signals, and these trigger points are used for retrospective cine reconstruction. The proposed approach is compared with ECG-gated ATR-SSFP and balanced steady-state free precession (bSSFP) in ten volunteers and five patients.

Results-The difference of ECG and self-gating trigger times has a variability of $13 \pm 11 \mathrm{~ms}$ (mean $\pm \mathrm{SD}$ ). Qualitative reviewer scoring and ranking indicate no statistically significant differences $(P>0.05)$ between self-gated and ECG-gated ATR-SSFP images. Quantitative bloodmyocardial border sharpness is not significantly different among self-gated ATR-SSFP $(0.61 \pm$ $\left.0.15 \mathrm{~mm}^{-1}\right)$, ECG-gated ATR-SSFP $\left(0.61 \pm 0.15 \mathrm{~mm}^{-1}\right)$, or conventional ECG-gated bSSFP cine $\operatorname{MRI}\left(0.59 \pm 0.15 \mathrm{~mm}^{-1}\right)$.

Conclusion-The proposed self-gated ATR-SSFP sequence enables fat-suppressed cardiac cine imaging at $1.5 \mathrm{~T}$ without the need for ECG gating and without decreasing the imaging efficiency of ATR-SSFP.

\section{Keywords}

self-gating; cardiac imaging; cine MRI; ATR; SSFP

Address correspondence to: Reeve Ingle, M.S., Packard Electrical Engineering Bldg, Room 210, 350 Serra Mall, Stanford, CA 94305-9510, TEL: (650) 723-3810, FAX: (650) 723-8473, ringle@ stanford.edu. 


\section{Introduction}

Magnetic resonance (MR) cardiac cine imaging with balanced steady-state free precession (bSSFP) is widely accepted as the gold standard for assessment of ventricular function (1). A segmented, retrospectively gated approach is commonly used, in which an electrocardiogram (ECG) signal is recorded and used to sort or interpolate the acquired data into different cardiac phases. The need for external ECG monitoring can lead to prolonged patient preparation times, triggering errors due to interference from gradient and radiofrequency $(\mathrm{RF})$ fields $(2,3)$, and triggering inconsistencies due to the magnetohydrodynamic effect. Some of these drawbacks can be addressed with techniques such as acoustic triggering (4) or pulse oximetry, both of which rely on alternative hardware systems to monitor the cardiac cycle. Alternatively, self-gated approaches have been developed to derive cardiac phase information directly from acquired MR data (5-16). These approaches require no additional hardware, simplifying patient preparation and improving protocol efficiency.

The bSSFP pulse sequence is widely used for cardiac cine imaging at $1.5 \mathrm{~T}$ due to its high signal-to-noise ratio (SNR) efficiency and excellent blood-myocardial image contrast (1719). However, bSSFP yields bright water and fat signals, which can make it difficult to distinguish pericardial fluid from fat, hinder the characterization of lipomas and other tumors, and generate fat-water cancellation artifacts that obscure vessels and other fat-water interfaces. Because of these limitations, separate pulse sequences with $T_{1}$ weighting, fat saturation preparation, or chemical-shift-based water-fat decomposition are often acquired in addition to the standard bSSFP cine acquisitions. Several recent works have applied waterfat separation techniques to bSSFP cardiac cine imaging acquisitions, enabling discrimination of these features using a single pulse sequence (20,21). Drawbacks of these techniques include lengthened repetition times (TRs) and poorer temporal resolution due to multi-echo acquisitions (20) or sensitivity to $B_{0}$ inhomogeneity and partial-volume limitations due to the phase-based discrimination of water and fat (21).

In certain clinical applications, suppression of the bright fat signal may be sufficient for diagnostic purposes and could potentially reduce chemical shift and motion artifacts compared to standard bSSFP cine imaging. Lin, et al., proposed an alternating repetition time (ATR) bSSFP sequence for fat-suppressed cardiac cine imaging at $1.5 \mathrm{~T}$ (22). ATRSSFP yields steady-state fat suppression at $1.5 \mathrm{~T}$ through the addition of a short 1.15 -ms TR $\left(\mathrm{TR}_{2}\right)$ after the imaging $\mathrm{TR}\left(\mathrm{TR}_{1}\right)$ (23). Because the available acquisition time during $\mathrm{TR}_{2}$ is very short, it is not typically used for imaging purposes, making ATR-SSFP slightly less time efficient than bSSFP. Despite this small time penalty, the fat suppression requirements of many cardiac imaging applications, such as coronary MR angiography, have made ATRSSFP an attractive alternative to $\operatorname{bSSFP}(22,24,25)$.

Wideband SSFP is another alternating repetition time sequence that uses a longer $\mathrm{TR}_{2}$ and different phase cycling scheme to increase the bSSFP band spacing for banding artifact reduction (26). This sequence has been used for cardiac cine imaging at $3 \mathrm{~T}$ (27), and the authors demonstrated that the lengthened $\mathrm{TR}_{2}$ could be used to acquire self-navigation data for respiratory-gated free-breathing cine imaging (28).

Magn Reson Med. Author manuscript; available in PMC 2016 May 01. 
In this work, we develop a self-gated ATR-SSFP pulse sequence for fat-suppressed cine imaging at $1.5 \mathrm{~T}$. By bridging the slice-select rephasing gradients of an ATR-SSFP pulse sequence, we enable the acquisition of a one-dimensional (1D) projection self-gating signal during the unused $1.15-\mathrm{ms} \mathrm{TR}_{2}$ interval. This self-gating signal is used for retrospectively gated reconstruction of breath-held segmented cine acquisitions. The proposed sequence is used to image ten healthy volunteers and five patients for comparison with ECG-gated bSSFP and ATR-SSFP acquisitions. Cardiac trigger times derived from the self-gating signal are compared to recorded ECG R-wave triggers to evaluate the accuracy of the proposed self-gating technique. ATR-SSFP images are retrospectively reconstructed using both ECG and self-gating trigger times for comparison of image quality between the two techniques. The proposed sequence enables fat-suppressed cardiac cine imaging without the need of ECG or other external devices to monitor the cardiac cycle.

\section{Methods Pulse Sequence}

The fat-suppressed ATR-SSFP pulse sequence proposed by Leupold, et al. (23), was slightly modified in this work to enable the acquisition of a 36-point 1D projection self-gating signal during the unused $\mathrm{TR}_{2}$. The slice-select rephasing and prephasing gradient lobes were bridged to yield a constant-amplitude gradient during $\mathrm{TR}_{2}$ having the same net area for fully refocused imaging. Figure 1a shows the proposed self-gated ATR-SSFP pulse sequence used in this work. A hamming-windowed sinc RF pulse with time-bandwidth product of 1.5 and pulse duration of $500 \mu$ s was used. Additional time allotment was required before and after each RF pulse for RF amplifier unblanking, transmit frequency and phase initialization, and receive coil detuning, leaving approximately $144 \mu$ s during $\mathrm{TR}_{2}$ for acquisition of a 36point self-gating readout along $k_{z}$. Due to the limited acquisition time and the gradient amplitude requirements for fully refocused imaging, the spatial resolution of each 1D selfgating acquisition was limited to approximately $8.5 \mathrm{~mm}$. Figure $1 \mathrm{~b}$ shows the ATR-SSFP spectral profile within $\mathrm{TR}_{1}$ and $\mathrm{TR}_{2}$. The steady-state signal within $\mathrm{TR}_{1}$ contains a null on the fat resonance at $1.5 \mathrm{~T}$, while the signal within $\mathrm{TR}_{2}$ is very large for both water and fat resonances.

Retrospectively gated bSSFP and ATR-SSFP cine imaging was performed during separate end-expiratory breath holds. A 2D Cartesian readout and linear $k$-space segmentation with 12 views per segment were used, yielding temporal resolutions of $43.2 \mathrm{~ms}$ ( $\mathrm{TR}=3.6 \mathrm{~ms}$ ) for $\mathrm{bSSFP}$ and $57.6 \mathrm{~ms}\left(\mathrm{TR}_{1} / \mathrm{TR}_{2}=3.65 / 1.15 \mathrm{~ms}\right)$ for ATR-SSFP. All other imaging parameters were matched for bSSFP and ATR-SSFP acquisitions: field of view $=30 \times 30$ $\mathrm{cm}^{2}$, resolution $=1.5 \times 1.9 \mathrm{~mm}^{2}$, acquisition matrix size $=200 \times 156$, reconstructed frames $=30$, ip angle $=50^{\circ}$, and slice thickness $=6 \mathrm{~mm}$. To reach steady state, a 40-TR linear catalyzation train was used followed by 460 dummy views.

Acquisitions were not synchronized to the cardiac cycle, and each $k$-space segment was repeated for a duration spanning 1.15 times the expected R-to-R (RR) interval to sample the entire cardiac cycle. A vector electrocardiogram signal was acquired during each scan, and the ECG trigger time stamps provided by the scanner's physiological monitoring system were recorded and used for retrospectively gated reconstruction. Self-gating data were also 
acquired during $\mathrm{TR}_{2}$ for all ATR-SSFP scans and used to derive cardiac trigger points for retrospective self-gated reconstruction. In this manner, each ATR-SSFP acquisition was reconstructed using retrospective ECG gating and self gating for direct comparison of the two techniques.

\section{Self-Gating Signal Processing}

Self-gating signal processing and retrospectively gated image reconstruction were conducted off-line in MATLAB (MathWorks, Natick, MA). Each self-gating acquisition was first summed along $k_{z}$ to yield a single-point projection signal from the center of the excited slice. Summing the complex-valued self-gating data along $k_{z}$ is equivalent to extracting the central point from the 1D discrete Fourier transform (DFT). Since the self-gating readout is acquired along $k_{z}$, the 1D DFT represents a projection of the excited slice in the throughslice direction. Summing the acquired self-gating data yields a complex time-varying waveform from each receive coil. Each waveform contains cyclic amplitude and phase variations due to changing motion and flow patterns within the cardiac cycle, and the differing coil sensitivity profiles lead to unique self-gating waveforms for each receive coil. The time-varying bipolar phase-encoding gradients in $\mathrm{TR}_{1}$ also induce undesired oscillations and variability in the self-gating signals due to eddy currents, gradient hysteresis, and flow encoding. While the segmented linear phase-encode ordering used in this work minimizes eddy-current-induced image artifacts (29), it yields coherent flow-related perturbations of the self-gating signals. To mitigate these issues, bandpass filtering and template matching were used to derive robust cardiac trigger points from these self-gating signals.

Self-gating signals from each channel were bandpass filtered using a Kaiser-windowed finite impulse response (FIR) filter of order 500 and $\beta=4.5$. Low and high cutoff frequencies were $0.6 \mathrm{~Hz}$ and $10 \mathrm{~Hz}$, respectively, which were chosen conservatively to ensure very little attenuation throughout the range of expected in-vivo heart rates. Bandpass filtering was performed in both the forward and reverse directions to yield zero group delay. Bandpass filtering removes the DC offset and low-frequency variations such as baseline drift due to imperfect breath holds, and it also removes high-frequency oscillations from eddy currents and other noise sources. Figure 2 shows the self-gating signal from one coil of a representative four-chamber breath-held scan. The raw self-gating data (Fig. 2a) are summed along $k_{z}$ (Fig. 2b) and then bandpass filtered (Fig. 2c). Note the periodic pattern that matches the cardiac cycle.

Close inspection of Fig. $2 \mathrm{c}$ also reveals a slow change in shape of the underlying cyclic pattern from beginning to end of the acquisition, which is most apparent in the real part of the self-gating signal. This signal contains sharp spikes during systole due to high flow. The amplitude of these spikes is smallest in the middle of the acquisition, where the central phase-encode lines are acquired, and the polarity tends to reverse from beginning to end of the acquisition. This signal variation is most likely caused by flow encoding from the bipolar phase-encoding gradients, which reverse in polarity and change linearly throughout the acquisition. To address these coherent self-gating signal variations due to the linear phaseencode ordering, an adaptive template-matching technique was developed to derive robust cardiac trigger points from the filtered self-gating signals. Template matching was found to 
be superior to peak detection for derivation of self-gating trigger points (30). The templatematching technique developed for this work is similar to the method used in (30), but adapted to better address self-gating signal perturbations due to the linear phase-encode ordering. Rather than convolving the self-gating signals with a single median template as in (30), multiple templates are directly selected from the self-gating signals and used to derive a single trigger point immediately preceding each template location. This localized templatematching approach limits each trigger point calculation to a short time frame where the selfgating signals are most similar, and it better addresses slow perturbations that can be caused by the linear phase-encode ordering.

Template matching was performed using all channels of the bandpass-filtered self-gating signals. The template duration was set to half of the mean RR interval, which was determined by computing the Fourier transform of the self-gating signals and locating the largest magnitude peak between 40 and 100 cycles per minute. The initial template location and corresponding trigger point can be selected freely. To avoid filter rolloff effects at the end of the waveforms, we chose to work backward from the end of the self-gating signals, as the beginning of the acquisition was preceded by dummy views. Starting from the end of the self-gating signals, a template was extracted, and the cross correlation between the template and the self-gating signal was computed. This cross correlation was repeated for all channels, the results were summed, and peak detection was used to find the trigger point immediately preceding the template window location. This detected trigger point was used to update the template region, and the process was repeated to determine the preceding trigger point. This iterative process of template extraction, cross correlation, and trigger point detection was repeated to find all trigger points from the self-gating signal. The template-matching algorithm is illustrated in Fig. 3.

The resulting trigger points can have slight variations depending on the location of the first template region within the RR interval and the particular self-gating signal features that are included. To address this issue, the template-matching procedure was carried out 30 times using different starting template regions equally spaced within the mean RR interval. From the resulting 30 sets of candidate trigger times, the most common set was selected for the final self-gating trigger locations. Here, "most common" was determined by finding the set whose vector of RR intervals (first difference of trigger times) was closest (in a minimum root-mean-square sense) to the median vector of RR intervals among the 30 candidates. This vector-based comparison yields the most common set of trigger times among the 30 candidates, and it requires no assumption of RR-interval consistency throughout the acquisition.

\section{Image Reconstruction}

Retrospectively gated reconstruction was used to generate images at 30 cardiac phases evenly spaced throughout the mean RR interval. To facilitate direct comparison of ECGgated and self-gated ATR-SSFP reconstructions, the mean trigger delay (difference of selfgating trigger times and ECG R-wave trigger times) (8) was subtracted from the self-gating trigger times so that reconstructed images corresponded to the same cardiac phase. For each reconstruction, a time stamp indicating the time to the previous trigger point was computed 
for every TR of imaging data. To account for heart rate variations, these time stamps were normalized to the mean RR interval by expanding or contracting a single mid-diastolic time point using a soft-thresholding operation. Acquired data were then separated into respective phase-encode locations and sorted by these time stamps. For each phase encode, linear interpolation was used to compute $k$-space data at 30 time frames evenly spaced throughout the mean RR interval. Each frame of $k$-space data was multiplied by a 2D Tukey window to reduce Gibbs ringing artifacts and then zero padded to a matrix size of $512 \times 512$. Finally, a $2 \mathrm{D}$ fast Fourier transform (FFT) was performed to generate an image at each cardiac phase.

\section{In-Vivo Studies}

Imaging was performed on a 1.5 T GE Signa Twinspeed scanner (GE Healthcare,

Waukesha, WI, USA) with an eight-channel cardiac array coil. Pulse sequencing, scan control, and preliminary image reconstruction were performed using the RTHawk real-time environment (31) (HeartVista, Inc., Menlo Park, CA, USA). Images were obtained in ten healthy volunteers (six males, four females) and five patients (four males, one female) undergoing clinical cardiac MR (CMR) examinations. For the volunteer studies, a real-time spiral GRE scout sequence was used to prescribe three short-axis (SAX) scan planes (apical, middle, and basal slice locations) and one four-chamber $(4 \mathrm{CH})$, one three-chamber $(3 \mathrm{CH})$, and one two-chamber $(2 \mathrm{CH})$ scan plane. For each of the six scan planes, a 2D single-shot spiral field map was acquired, and a first-order polynomial fit within a region covering the heart was used to set the scanner center frequency and linear shim values. This field-mapbased shimming was performed automatically in RTHawk. For each scan plane, shimming was immediately followed by breath-held ATR-SSFP and bSSFP scans. Patient studies were conducted at the end of the clinical examination, and due to scan time limitations, a subset of the six scan planes was acquired for each patient. All patient studies were conducted 1030 minutes after intravenous injection of Gd-BOPTA. Prior to imaging, informed consent was obtained from all individuals, and the study was approved by our institutional review board (IRB).

\section{Temporal Variability Analysis}

Self-gating trigger times were compared to ECG trigger times by computing the trigger variability for each breath-held acquisition (8). Trigger variability measures the standard deviation of the trigger delay values and indicates the amount of temporal "jitter" between the self-gating and ECG trigger times. The trigger variability (TV) between a set of selfgating trigger points (S) and a set of ECG R-wave trigger points $(\mathbf{R})$ is given by

$$
T V=\sqrt{\frac{1}{N-1} \sum_{n=1}^{N}\left(\left(\mathrm{~S}_{n}-\mathrm{R}_{n}\right)-M T D\right)^{2}}, \quad \text { [1] }
$$

where $N$ is the total number of cardiac cycles and $M T D$ is the mean trigger delay,

$$
M T D=\frac{1}{N} \sum_{n=1}^{N}\left(\mathrm{~S}_{n}-\mathrm{R}_{n}\right)
$$


A nonzero MTD indicates an offset between all ECG triggers and self-gating triggers, due to triggering at different locations within the cardiac cycle. With nonzero MTD, the resulting self-gated and ECG-gated cine reconstructions will begin at different phases of the cardiac cycle. For this reason, the mean trigger delay was subtracted from the self-gating trigger times to synchronize the reconstructions and enable direct phase-by-phase comparison.

\section{Image Analysis}

Each ATR-SSFP acquisition was reconstructed using both ECG gating and self gating, and the resulting cine loops were displayed side by side in OsiriX (Pixmeo, Geneva, Switzerland) and played synchronously at 15 frames per second. The placement of ECGgated and self-gated reconstructions was randomized, and acquisitions from all ten volunteer and five patient studies were displayed in a randomized order. Two board-certified cardiologists independently assigned absolute image quality scores as well as integer rank scores to the ECG-gated and self-gated reconstructions. A total of 69 image pairs were scored by each reader. The following scoring system, which has been used in previous selfgated MRI studies $(7-9,30)$, was used to assign image quality scores: 4 points $=$ excellent image quality and definition of fine anatomic structures; 3 points $=$ good image quality, permitting identification of fine anatomic structures; 2 points = image quality adequate for interpretation; and 1 point = poor image quality, precluding adequate visualization of structures for interpretation. To better differentiate subtle differences between each ECGgated and self-gated cine reconstruction, the readers were also instructed to assign integer rank scores to each reconstruction $(-1=$ worst; $0=$ tie; $1=$ best $)$, with the possibility for ties when both cine reconstructions were considered to be of the same quality. Wilcoxon signedrank tests were used to analyze both image quality scores and image rank scores assigned by the two readers. The tests were used to assess the null hypothesis that differences between scores given to ECG-gated and self-gated reconstructions are centered about zero. A statistical significance level of $P=0.05$ was chosen for rejecting the null hypothesis.

Quantitative blood-myocardial sharpness measurements were computed for comparison of self-gated ATR-SSFP, ECG-gated ATR-SSFP, and ECG-gated bSSFP images using the same sharpness metric as previous free-breathing cardiac cine studies $(32,33)$. The distance between the $20 \%$ and $80 \%$ intensity points was measured on a line profile across the leftventricular blood-myocardial interface using the same location and the same mid-diastolic phase for the three cine techniques. The inverse of this distance was used as a measurement of sharpness. A two-tailed paired $t$-test was used for statistical evaluation.

Signal amplitudes were measured in the myocardium and epicardial fat in all bSSFP and self-gated ATR-SSFP cine datasets. Measurements were performed in a single end-diastolic frame from each cine series. Circular regions of interest were manually placed within the left ventricular myocardium and epicardial fat to measure mean signal amplitudes. Similar anatomical regions were selected in all images. The ratio of epicardial fat signal to myocardial signal was computed to compare the level of epicardial fat suppression of ATRSSFP to that of bSSFP (22). A two-tailed paired $t$-test was used to determine if there was a significant difference between bSSFP and ATR-SSFP signal ratios. 


\section{Results}

The imaging protocol was successfully carried out in ten healthy volunteers and five patients. Among all volunteer and patient scans, the mean heart rate per scan ranged from 50-90 beats per minute (bpm), and the breath hold duration ranged from 13.6-20.7 seconds.

\section{Temporal Variability Analysis}

Filtered self-gating signals, self-gating trigger points, and ECG R-wave trigger points are shown in Fig. 4 for four different scan planes from a representative volunteer study. Among the four scan geometries shown in Fig. 4, the SAX self-gating signal has the highest degree of periodicity and the lowest $T V(3 \mathrm{~ms})$, indicating very little temporal variability (less than the imaging TR) between the ECG and self-gating trigger points. The long-axis $4 \mathrm{CH}, 3 \mathrm{CH}$, and $2 \mathrm{CH}$ self-gating signals all have cyclic patterns that vary coherently from beginning to the end of the acquisition. These variations can be seen in Fig. 4 as a slow change in amplitude and shape of the self-gating signal throughout the duration of each long-axisacquisition. Despite these underlying perturbations, the template-matching technique yields trigger points that are in good agreement with the ECG trigger points, with $T V=11 \mathrm{~ms}$ $(4 \mathrm{CH}), 19 \mathrm{~ms}(3 \mathrm{CH})$, and $12 \mathrm{~ms}(2 \mathrm{CH})$.

Among all studies, the mean $T V$ was $12.9 \mathrm{~ms}$, and the standard deviation was $11.4 \mathrm{~ms}$. The trends highlighted in Fig. 4, with SAX self-gating signals having improved periodicity and lower $T V$, held for the majority of studies. The mean $T V$ values among $\mathrm{SAX}, 4 \mathrm{CH}, 3 \mathrm{CH}$, and $2 \mathrm{CH}$ scan planes were $7.9 \pm 5.8 \mathrm{~ms}, 20.2 \pm 12.3 \mathrm{~ms}, 17.7 \pm 15.5 \mathrm{~ms}$, and $18.2 \pm 13.7 \mathrm{~ms}$, respectively. For all studies, $T V$ was less than the 58-ms temporal resolution of the ATRSSFP acquisition.

\section{ATR-SSFP Cine Images}

Representative ATR-SSFP images from a single volunteer study are shown in Fig. 5.

Systolic and diastolic images from SAX, 4CH, 3CH, and 2CH scans are shown in Fig. 5a-d, respectively. Images reconstructed with self gating are in very close agreement with images reconstructed with ECG gating, and the difference images show very few coherent differences between the two reconstructions. The SAX difference images in Fig. 5a contain less energy than the long-axis difference images in Fig. 5b-d, indicating better agreement between ECG and self-gating reconstructions for the SAX scan plane.

ECG-gated and self-gated ATR-SSFP images from two different patient studies are shown in Fig. 6. Figure 6a shows systolic and diastolic SAX and $4 \mathrm{CH}$ images for a patient with an apical septal infarct. Self-gated reconstructions are in close agreement with ECG-gated reconstructions, and the difference images are small and contain few coherent structures. The bright signal surrounding the left and right ventricles is not epicardial fat, but, rather, excess pericardial fluid indicating a small pericardial effusion. ATR-SSFP imaging was performed approximately 30 minutes after injection of Gd-BOPTA. Interestingly, regions of signal enhancement in the interventricular septal wall and apex are visible in the 4CH ATRSSFP images (black arrows). Due to suppression of epicardial fat signal, these regions could 
be better visualized in ATR-SSFP images than in bSSFP. These regions corresponded to a myocardial infarction seen with clinical delayed enhancement imaging.

Figure $6 \mathrm{~b}$ shows systolic and diastolic SAX and $4 \mathrm{CH}$ images for a patient with aortic valve replacement. Self-gated reconstructions are in close agreement with ECG-gated reconstructions, and the difference images are small and contain few coherent structures. ATR-SSFP imaging was performed following intravenous injection of Gd-BOPTA, and therefore the blood-myocardium contrast is reduced compared to the non-contrast-enhanced volunteer images in Fig. 5. The signal void in the left atrium is due to off-resonance shifts near the prosthetic aortic valve (black arrowheads), and the signal void in the anterior chest wall is due to off-resonance shifts from the wire sutures (white arrowheads).

\section{Image Analysis}

The mean and standard deviation of all image quality scores and image ranks assigned by the two reviewers are summarized in Table 1 . There were no statistically significant differences between image quality scores assigned to ECG-gated and self-gated ATR-SSFP reconstructions $(P=0.81)$, and the mean quality scores were essentially equivalent for the two techniques. Likewise, there was a minimal, non-significant difference in ranking scores between the ECG-gated and self-gated reconstructions $(P=0.14)$.

Among all datasets, the quantitative blood-myocardial border sharpness measurements were $0.61 \pm 0.15 \mathrm{~mm}^{-1}$ (self-gated ATR-SSFP), $0.61 \pm 0.15 \mathrm{~mm}^{-1}$ (ECG-gated ATR-SSFP), and $0.59 \pm 0.15 \mathrm{~mm}^{-1}$ (ECG-gated bSSFP). There were no statistically significant differences $(P$ $>0.05$ ) between the sharpness measurements of any of the three techniques.

\section{ATR-SSFP and bSSFP Image Comparison}

Self-gated ATR-SSFP images and ECG-gated bSSFP images are shown for three different volunteer studies in Fig. 7. ATR-SSFP achieved good suppression of signal from epicardial fat, while the level of fat suppression in the chest wall was variable due to off-resonance shifts. In bSSFP images, partial volume effects led to fat-water cancellation artifacts near the coronary arteries and right ventricular wall, while the fat-suppressed ATR-SSFP images enabled better visualization of these features (e.g., Fig. 7a,c). Ghosting artifacts from vessels with high flow were slightly worse for ATR-SSFP than bSSFP (e.g., Fig. 7b, bracketed region). Among all datasets, the measured fat-myocardium signal ratio reduced from $2.7 \pm$ 0.9 for bSSFP to $0.7 \pm 0.3$ for self-gated ATR-SSFP $(P<0.001)$.

\section{Discussion}

The proposed self-gated ATR-SSFP sequence enabled acquisition of self-gating signals during the unused $\mathrm{TR}_{2}$ of a fat-suppressed ATR-SSFP pulse sequence. In volunteer and patient studies, ATR-SSFP images reconstructed with the proposed self-gating technique were in very close agreement with ECG-gated reconstructions, having no statistically significant differences in image quality scores or image rankings. The ATR-SSFP sequence achieved good suppression of epicardial fat. Compared to bSSFP, partial volume effects that cause fat-water cancellation were significantly reduced with the ATR-SSFP sequence. 
Mean $T V$ values were comparable to those measured in other self-gated imaging studies (8, 9,11,15,16,30). The mean $T V$ for short-axis scan planes was less than that of long-axis scan planes, consistent with findings by Nijm, et al. (30). Likewise, short-axis self-gating signals typically had less variability from beginning to end of the acquisition. The magnitude and phase of the self-gating signal varies throughout the cardiac cycle due to factors such as changes in through-plane and in-plane flow and fluctuations in mean signal intensity within the imaged slice (e.g., from variations in blood-pool volumes). For short-axis acquisitions, the primary flow direction is through-plane, aligned with the self-gating readout axis $\left(k_{z}\right)$. For long-axis acquisitions, flow directions are more complex, often having a large component aligned with the flow-sensitive phase-encode direction. Therefore, undesired flow-related perturbations of the self-gating signals are typically more pronounced for longaxis scans than short-axis scans, as seen in Fig. 4.

To account for these flow-related self-gating signal perturbations, a localized templatematching algorithm was developed. As illustrated in Fig. 3, short template regions were selected directly from the self-gating signal and used to derive adjacent trigger points. This pattern detection technique utilizes the high correlation within temporally localized segments, and it is insensitive to slow perturbations that may affect the overall periodicity of the signal. Additionally, the self-gating signals from most of the eight receiver coils typically exhibit some degree of periodicity that is correlated with the cardiac cycle. Each coil is sensitive to different regions of the heart, aorta, and other anatomical structures that have different cardiac-related signal and flow fluctuations. Thus, the self-gating signals from each coil are unique, but they exhibit the same underlying cardiac-induced periodicity. By utilizing the self-gating signals from all coils, the template-matching algorithm uses all of the available information about the cardiac cycle for robust trigger detection.

Although single-coil trigger detection methods are commonly used for self-gated cardiac cine imaging, recent multi-coil approaches for cardiac and respiratory self-gated cine imaging have had success and eliminate some of the challenges involved with coil selection $(33,34)$. While we found that manually selecting a single coil for template matching led to lower (better) $T V$ in some datasets, the specific coil or coils that outperformed the multi-coil technique used in this work varied between subjects and scan geometries. Likewise, in many datasets, there existed coils for which single-coil template matching yielded a higher (worse) $T V$. The proposed technique used self-gating signals from all coils, which led to robust trigger detection without the need for manual selection of a single "best" coil and resulted in a fully automated self-gated reconstruction process.

Several alternatives exist to address the flow-related issues that led to higher $T V$ and higher variability for long-axis scans. Pulse sequence modifications can be implemented to minimize or eliminate these sources of flow-related self-gating signal variability. One such technique is to use a different phase-encode ordering to better randomize the flow sensitivity throughout the entire duration of the acquisition. However, this randomization comes at the expense of increased eddy-current artifacts due to larger phase-encode jumps (29). Another alternative is to use a flow-insensitive imaging acquisition, such as $2 \mathrm{D}$ projection reconstruction. In this work, we chose to use a 2D Cartesian acquisition with linear phaseencode segmentation due to its widespread clinical use and general robustness to systematic 
errors such as eddy currents and gradient delays. However, the self-gated ATR-SSFP sequence and trigger detection algorithm presented here are compatible with other phaseencode orderings and imaging trajectories.

A limitation of many self-gated cine imaging techniques, including the proposed technique, is that triggering can occur at arbitrary locations in the cardiac cycle, such that different reconstructed cine datasets begin at different cardiac phases. Because cine images are typically viewed in a repeated loop, the starting phase of each loop is not a significant issue for slice-by-slice display. However, in applications requiring synchronization of cine frames, such as the simultaneous display of multiple slices or automated image processing techniques, additional steps will be required to translate the detected self-gating trigger points to a specific reference point in the cardiac cycle. This synchronization could be performed manually by looking at the reconstructed images, determining the offset of the starting frame with respect to the desired cardiac phase, and subtracting this offset from all self-gating trigger points. The offset time could also be determined through automatic image processing techniques that detect specific features within the cardiac cycle, such as minimal left ventricular volume or closure of a valve (16).

A limitation of this study is that no imaging was performed in subjects with arrhythmias. Modifications to the template-matching algorithm may be required to address cases of arrhythmia, specifically if the features of the self-gating signal are significantly different for normal and arrhythmic heart beats. Furthermore, modifications to the image reconstruction algorithm will be required to appropriately reject or modify imaging data from arrhythmic heart beats, and additional oversampling and/or iterative reconstruction techniques could be required to avoid undersampling artifacts. Alternatively, real-time processing of the selfgating data could be used to detect arrhythmias and reacquire necessary imaging data before the end of the scan. The filtering and template-matching techniques used in this work are limited to retrospective reconstruction, and alternative techniques, such as Kalman filtering (35), would be necessary for such a prospective approach. The modifications and analysis required to address arrhythmias are not trivial, and this work has been left as an area for future research.

Balanced steady-state pulse sequences such as bSSFP and ATR-SSFP are sensitive to offresonance shifts, which can lead to banding artifacts. Additionally, off-resonance can lead to variable fat suppression for ATR-SSFP due to the relatively narrow stopband centered on the fat resonance (Fig. 1b). We found that the targeted shimming approach used in this study was sufficient to yield robust suppression of epicardial fat and minimal banding within the heart. Banding artifacts and variable fat suppression were often present in the chest wall, liver, and other regions outside the heart (e.g., Fig. 7). Without targeted shimming or robust center frequency detection, the degree of epicardial fat suppression afforded by ATR-SSFP could be more variable. For imaging at field strengths other than $1.5 \mathrm{~T}$, modifications to the ATR-SSFP pulse sequence will be necessary for fat suppression due to the change in chemical shift. ATR sequence modifications have been proposed for fat-suppressed imaging at $3 \mathrm{~T}(23,36)$. However, additional banding-reduction measures may be required due to poorer field homogeneity at higher field strengths (37).

Magn Reson Med. Author manuscript; available in PMC 2016 May 01. 
Compared to bSSFP, we noticed a slight increase in flow artifacts in ATR-SSFP images. These flow artifacts were typically isolated to systolic phases and located near vessels with particularly high flow (e.g., Fig. 7b). Another drawback of ATR-SSFP compared to bSSFP is that the longer TR ( $4.8 \mathrm{~ms}$ vs. $3.6 \mathrm{~ms}$, respectively) requires longer breath holds or poorer temporal resolution.

The self-gated ATR-SSFP sequence presented in this work is not limited to breath-held 2D Cartesian cine imaging. Future work will explore non-Cartesian imaging trajectories such as radial and spiral acquisitions, which have benefits such as improved imaging efficiency and reduced flow sensitivity. In these non-Cartesian applications, the fat suppression of ATRSSFP may help to reduce off-resonance artifacts. Future investigations will also focus on free-breathing 2D and 3D cine applications, in which the self-gating signals can be used to track respiratory motion throughout the scan $(32,38)$.

\section{Conclusion}

The proposed self-gated ATR-SSFP sequence has enabled robust fat-suppressed cardiac cine imaging without the need for external ECG gating. Bridging the slice-select rephasing gradients permitted acquisition of $1 \mathrm{D}$ self-gating signals during the unused $\mathrm{TR}_{2}$ interval of the ATR-SSFP pulse sequence. Therefore, self-gated ATR-SSFP required no reduction in scan efficiency compared with retrospective ECG-gated ATR-SSFP. In breath-held scans conducted in volunteers and patients, there was little variability between self-gated trigger points and ECG trigger points. Likewise, there were no statistically significant differences in image quality scores and image rankings between self-gated and ECG-gated cine reconstructions. Compared to bSSFP cine imaging, ATR-SSFP yielded robust suppression of epicardial fat, which reduced fat-water cancellation artifacts that can obscure the visualization of ventricular borders, coronary arteries, and other fat-water interfaces. In addition to ECG-free self-gated cine imaging, the proposed sequence should be well suited for respiratory motion tracking in free-breathing 2D and 3D cardiac cine applications.

\section{Acknowledgements}

The authors thank Brian Hargreaves, Holden Wu, Okai Addy, and Taehoon Shin for valuable discussions.

This work was supported by:

Grant sponsor: National Science Foundation (NSF) Graduate Research Fellowship; Grant number: DGE-0645962; Grant sponsor: National Defense Science and Engineering Graduate (NDSEG) Fellowship; Grant number: 32 CFR 168a; Grant sponsor: John and Kate Wakerly Stanford Graduate Fellowship; Grant sponsor: National Institutes of Health (NIH); Grant numbers: R01 HL039297 and R01 HL075803; Grant sponsor: GE Healthcare.

\section{References}

1. Manning, WJ.; Pennell, DJ. Cardiovascular magnetic resonance. 2nd ed.. Philadelphia, PA: Saunders/Elsevier; 2010.

2. Polson MJ, Barker AT, Gardiner S. The effect of rapid rise-time magnetic fields on the ECG of the rat. Clin Phys Physiol Meas. 1982; 3:231-234. [PubMed: 7140162]

3. Shetty AN. Suppression of radiofrequency interference in cardiac gated MRI: a simple design. Magn Reson Med. 1988; 8:84-88. [PubMed: 3173072] 
4. Becker M, Frauenrath T, Hezel F, Krombach GA, Kremer U, Koppers B, Butenweg C, Goemmel A, Utting JF, Schulz-Menger J, Niendorf T. Comparison of left ventricular function assessment using phonocardiogram- and electrocardiogram-triggered 2D SSFP CINE MR imaging at $1.5 \mathrm{~T}$ and $3.0 \mathrm{~T}$. Eur Radiol. 2010; 20:1344-1355. [PubMed: 20013275]

5. Spraggins TA. Wireless retrospective gating: application to cine cardiac imaging. Magn Reson Imaging. 1990; 8:675-681. [PubMed: 2266792]

6. Kim WS, Mun CW, Kim DJ, Cho ZH. Extraction of cardiac and respiratory motion cycles by use of projection data and its applications to NMR imaging. Magn Reson Med. 1990; 13:25-37. [PubMed: 2319933]

7. White RD, Paschal CB, Clampitt ME, Spraggins TA, Lenz GW. Electrocardiograph-independent, “wireless" cardiovascular cine MR imaging. J Magn Reson Imaging. 1991; 1:347-355. [PubMed: $1802148]$

8. Larson AC, White RD, Laub G, McVeigh ER, Li D, Simonetti OP. Self-gated cardiac cine MRI. Magn Reson Med. 2004; 51:93-102. [PubMed: 14705049]

9. Crowe ME, Larson AC, Zhang Q, Carr J, White RD, Li D, Simonetti OP. Automated rectilinear self-gated cardiac cine imaging. Magn Reson Med. 2004; 52:782-788. [PubMed: 15389958]

10. Uribe S, Muthurangu V, Boubertakh R, Schaeffter T, Razavi R, Hill DLG, Hansen MS. Wholeheart cine MRI using real-time respiratory self-gating. Magn Reson Med. 2007; 57:606-613. [PubMed: 17326164]

11. Buehrer M, Curcic J, Boesiger P, Kozerke S. Prospective self-gating for simultaneous compensation of cardiac and respiratory motion. Magn Reson Med. 2008; 60:683-690. [PubMed: 18727084]

12. Schulz J, Korn M, Deimling M, Semmler W, Bock M. Flow-compensated self-gating. MAGMA. 2008; 21:307-315. [PubMed: 18668271]

13. Odille F, Uribe S, Batchelor PG, Prieto C, Schaeffter T, Atkinson D. Model-based reconstruction for cardiac cine MRI without ECG or breath holding. Magn Reson Med. 2010; 63:1247-1257. [PubMed: 20432296]

14. Liu J, Spincemaille P, Codella NCF, Nguyen TD, Prince MR, Wang Y. Respiratory and cardiac self-gated free-breathing cardiac CINE imaging with multiecho 3D hybrid radial SSFP acquisition. Magn Reson Med. 2010; 63:1230-1237. [PubMed: 20432294]

15. Krämer M, Herrmann KH, Biermann J, Reichenbach J. Retrospective reconstruction of cardiac cine images from golden-ratio radial MRI using one-dimensional navigators. J Magn Reson Imaging. 2013

16. Kolbitsch C, Prieto C, Schaeffter T. Cardiac functional assessment without electrocar-diogram using physiological self-navigation. Magn Reson Med. 2014; 71:942-954.

17. Carr HY. Steady-state free precession in nuclear magnetic resonance. Phys Rev. 1958; 112:16931701.

18. Oppelt A, Graumann R, Barfuss H, Fischer H, Hartl W, Shajor W. FISP — a new fast MRI sequence. Electromedica. 1986; 54:15-18.

19. Carr JC, Simonetti O, Bundy J, Li D, Pereles S, Finn JP. Cine MR angiography of the heart with segmented true fast imaging with steady-state precession. Radiology. 2001; 219:828-834. [PubMed: 11376278]

20. Reeder SB, Markl M, Yu H, Hellinger JC, Herfkens RJ, Pelc NJ. Cardiac CINE imaging with IDEAL water-fat separation and steady-state free precession. J Magn Reson Imaging. 2005; 22:44-52. [PubMed: 15971192]

21. Goldfarb JW, Arnold-Anteraper S. Water-fat separation imaging of the heart with standard magnetic resonance bSSFP CINE imaging. Magn Reson Med. 2013

22. Lin HY, Raman SV, Chung YC, Simonetti OP. Rapid phase-modulated water excitation steadystate free precession for fat suppressed cine cardiovascular MR. J Cardiovasc Magn Reson. 2008; 10:22. [PubMed: 18477396]

23. Leupold J, Hennig J, Scheffer K. Alternating repetition time balanced steady state free precession. Magn Reson Med. 2006; 55:557-565. [PubMed: 16447171] 
24. Wu HH, Gurney PT, Hu BS, Nishimura DG, McConnell MV. Free-breathing multiphase wholeheart coronary MR angiography using image-based navigators and three-dimensional cones imaging. Magn Reson Med. 2013; 69:1083-1093. [PubMed: 22648856]

25. Ingle RR, Wu HH, Addy NO, Cheng JY, Yang PC, Hu BS, Nishimura DG. Nonrigid autofocus motion correction for coronary MR angiography with a 3D cones trajectory. Magn Reson Med. 2013

26. Nayak KS, Lee HL, Hargreaves BA, Hu BS. Wideband SSFP: alternating repetition time balanced steady state free precession with increased band spacing. Magn Reson Med. 2007; 58:931-938. [PubMed: 17969129]

27. Lee HL, Shankaranarayanan A, Pohost GM, Nayak KS. Improved 3-Tesla cardiac cine imaging using wideband. Magn Reson Med. 2010; 63:1716-1722. [PubMed: 20512877]

28. Lee, HL.; Shankaranarayanan, A.; Nayak, KS. Retrospective self-navigated cine imaging using the unused echo in alternating TR SSFP. Proceedings of the 17th Annual Meeting of ISMRM; Honolulu, USA. 2009. p. 4643

29. Bieri O, Markl M, Scheffer K. Analysis and compensation of eddy currents in balanced SSFP. Magn Reson Med. 2005; 54:129-137. [PubMed: 15968648]

30. Nijm GM, Sahakian AV, Swiryn S, Carr JC, Sheehan JJ, Larson AC. Comparison of self-gated cine MRI retrospective cardiac synchronization algorithms. J Magn Reson Imaging. 2008; 28:767772. [PubMed: 18777546]

31. Santos JM, Wright GA, Pauly JM. Flexible real-time magnetic resonance imaging framework. Conf Proc IEEE Eng Med Biol Soc. 2004; 2:1048-1051. [PubMed: 17271862]

32. Larson AC, Kellman P, Arai A, Hirsch GA, McVeigh E, Li D, Simonetti OP. Preliminary investigation of respiratory self-gating for free-breathing segmented cine MRI. Magn Reson Med. 2005; 53:159-168. [PubMed: 15690515]

33. Hu P, Hong S, Moghari MH, Goddu B, Goepfert L, Kissinger KV, Hauser TH, Manning WJ, Nezafat R. Motion correction using coil arrays (MOCCA) for free-breathing cardiac cine MRI. Magn Reson Med. 2011; 66:467-475. [PubMed: 21773986]

34. Han, F.; Stanislas, R.; Hu, P. Improved cardiac motion self-gating. Proceedings of the 21 st Annual Meeting of ISMRM; Salt Lake City, USA. 2013. p. 485

35. Spincemaille P, Nguyen TD, Prince MR, Wang Y. Kalman filtering for real-time navigator processing. Magn Reson Med. 2008; 60:158-168. [PubMed: 18581354]

36. Gonçalves SI, Ziech MLW, Lamerichs R, Stoker J, Nederveen AJ. Optimization of alternating TRSSFP for fat-suppression in abdominal images at 3T. Magn Reson Med. 2012; 67:595-600. [PubMed: 22189839]

37. Bangerter NK, Hargreaves BA, Vasanawala SS, Pauly JM, Gold GE, Nishimura DG. Analysis of multiple-acquisition SSFP. Magn Reson Med. 2004; 51:1038-1047. [PubMed: 15122688]

38. Brau ACS, Brittain JH. Generalized self-navigated motion detection technique: Preliminary investigation in abdominal imaging. Magn Reson Med. 2006; 55:263-270. [PubMed: 16408272] 

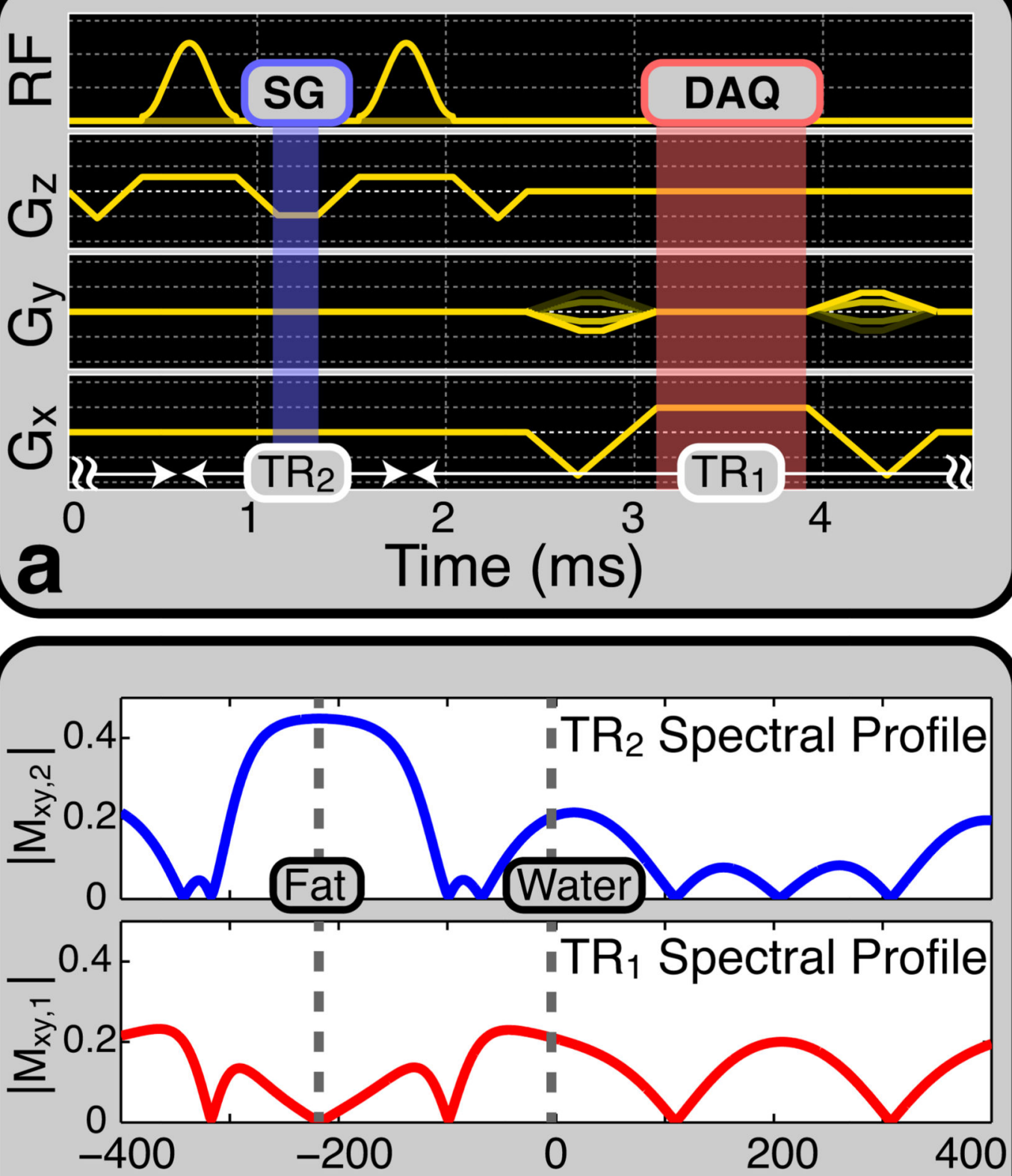

b Off-resonance frequency $(\mathrm{Hz})$

Figure 1.

Self-gated ATR-SSFP pulse sequence. (a) Bridging the slice-select rephasing lobes during $\mathrm{TR}_{2}$ enables acquisition of $1 \mathrm{D}$ self-gating (SG) readouts along $k_{z}$ with no time penalty compared to standard ATR-SSFP. (b) The ATR-SSFP spectral profile yields fat suppression during $\mathrm{TR}_{1}$ while maintaining high fat and water signal during $\mathrm{TR}_{2}$. 


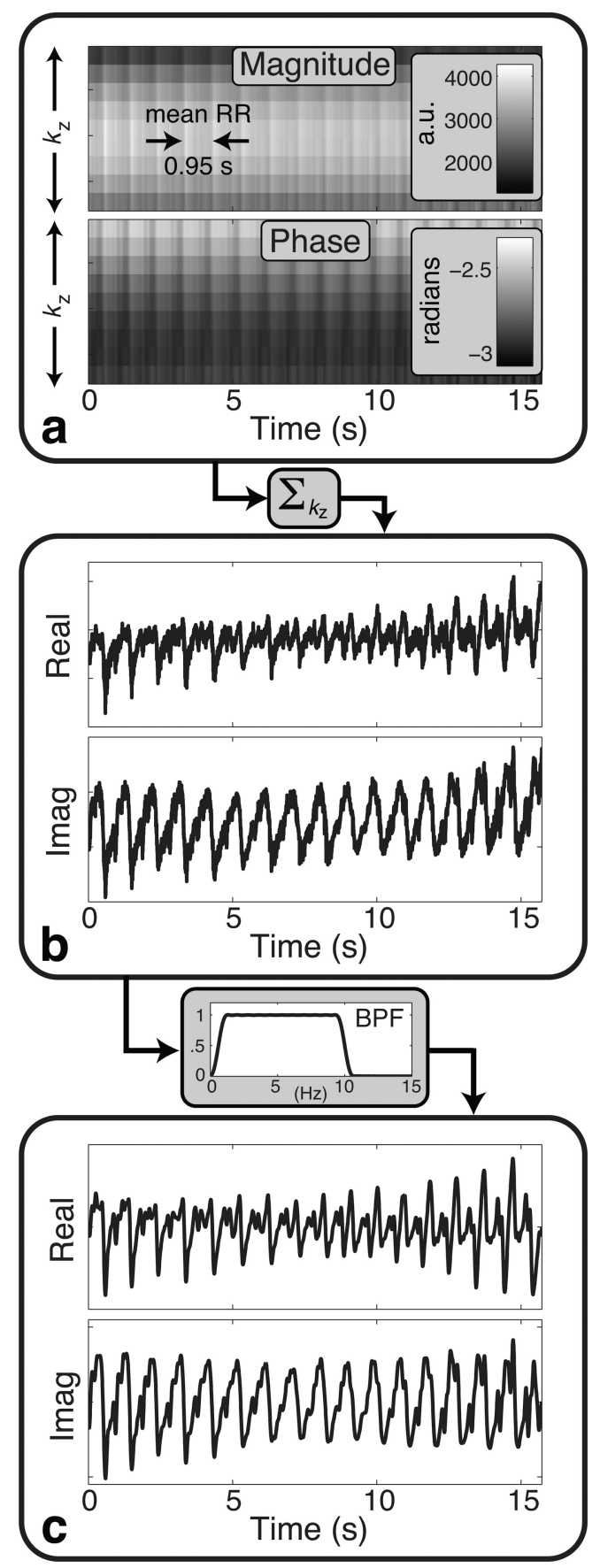

Figure 2.

Representative self-gating signal from a single anterior coil located near the heart. (a) Raw self-gating $k$-space data shows periodic uctuation with the cardiac cycle. (b) Summation along $k_{z}$ yields the self-gating signal from the center of the imaged slice. (c) Bandpass filtering (BPF) with a $0.6-10 \mathrm{~Hz}$ passband removes baseline drift and high-frequency noise from eddy currents and other unwanted sources. 


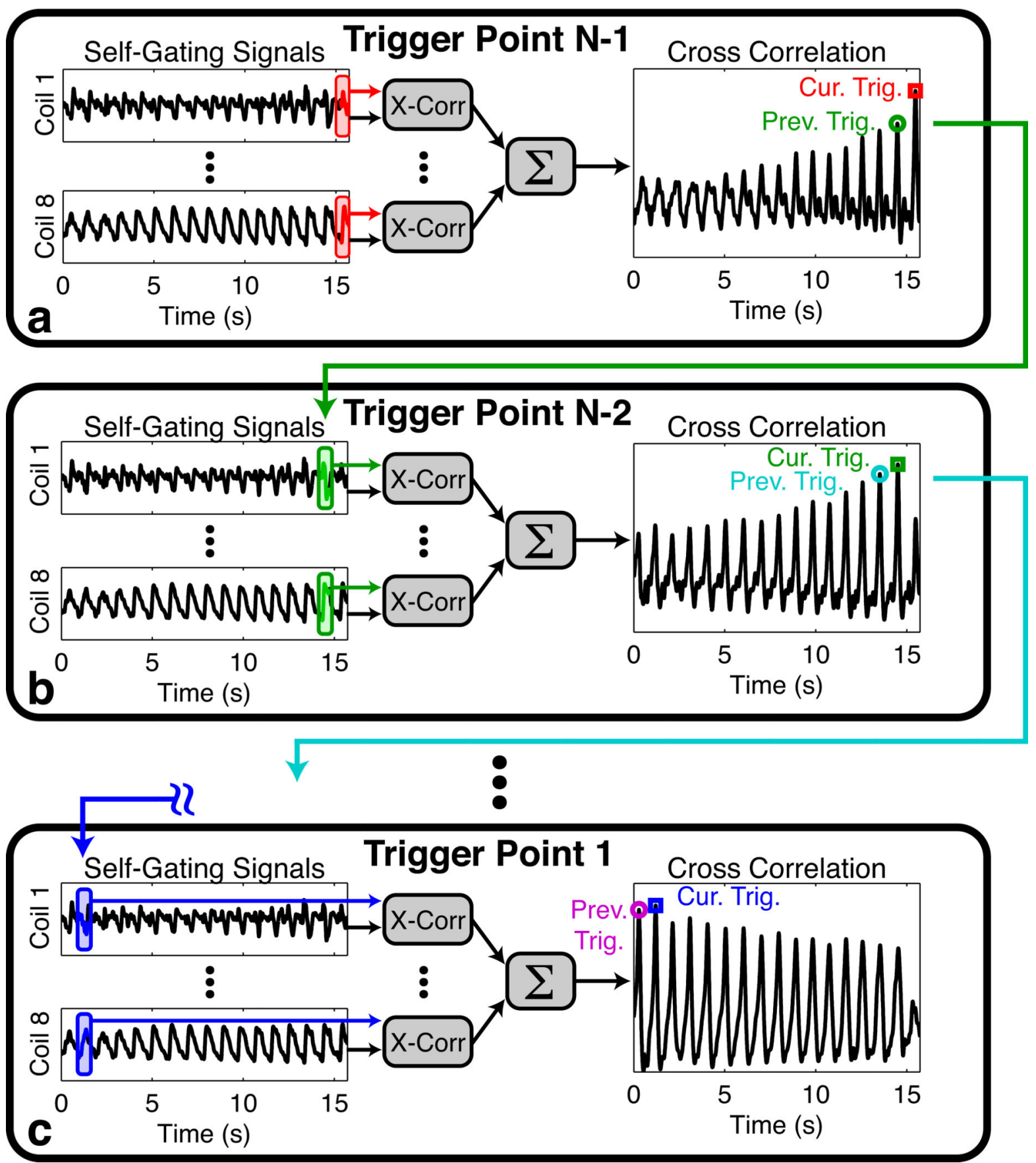

Figure 3.

Template-matching algorithm for self-gating trigger detection. (a) Working backward from the end of the self-gating signals, template regions (red rectangles) spanning half of the mean RR interval are extracted directly from the filtered self-gating signals (real part shown for coils 1 and 8). The cross correlation is computed between the template region and the rest of the corresponding self-gating signal. Templates are normalized to yield unity cross correlation for a relative shift of 0 . The results from all coils are summed to yield a crosscorrelation waveform, and peak detection is used to find the previous trigger point (green 
circle). (b) This trigger point is used to update the template regions (green rectangles), and the process is repeated to compute the previous trigger point (cyan circle). (c) This procedure is repeated to detect all trigger points, ending with the first template region (blue rectangle) and trigger point (purple circle). 

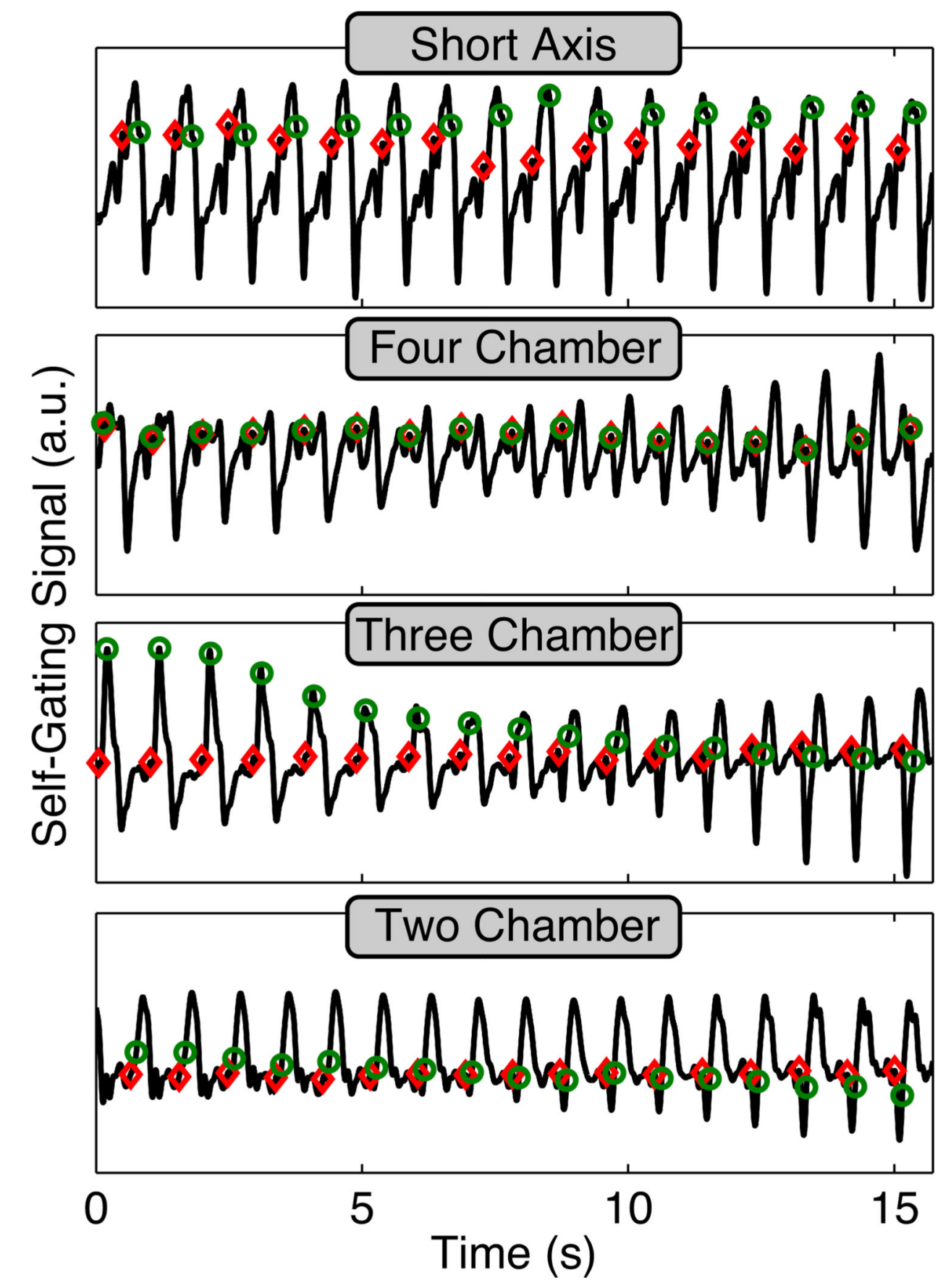

Figure 4.

Comparison of ECG R-wave trigger points and self-gating trigger points. Filtered self-gating signals (real part) from an anterior coil element of a representative volunteer study are shown for four different scan planes. ECG trigger points (red diamonds) and self-gating trigger points (green circles) are overlaid on each waveform. The SAX acquisition (top) has a highly periodic self-gating signal and the smallest trigger variability ( $3 \mathrm{~ms})$ among the four acquisitions. The long-axis acquisitions (bottom three rows) have self-gating signals with a slowly varying cyclic pattern from beginning to end of the acquisition. The trigger 
variabilities (11, 19, and $12 \mathrm{~ms}$ for $4 \mathrm{CH}, 3 \mathrm{CH}, 2 \mathrm{CH}$, respectively) are larger than that of the SAX acquisition. 

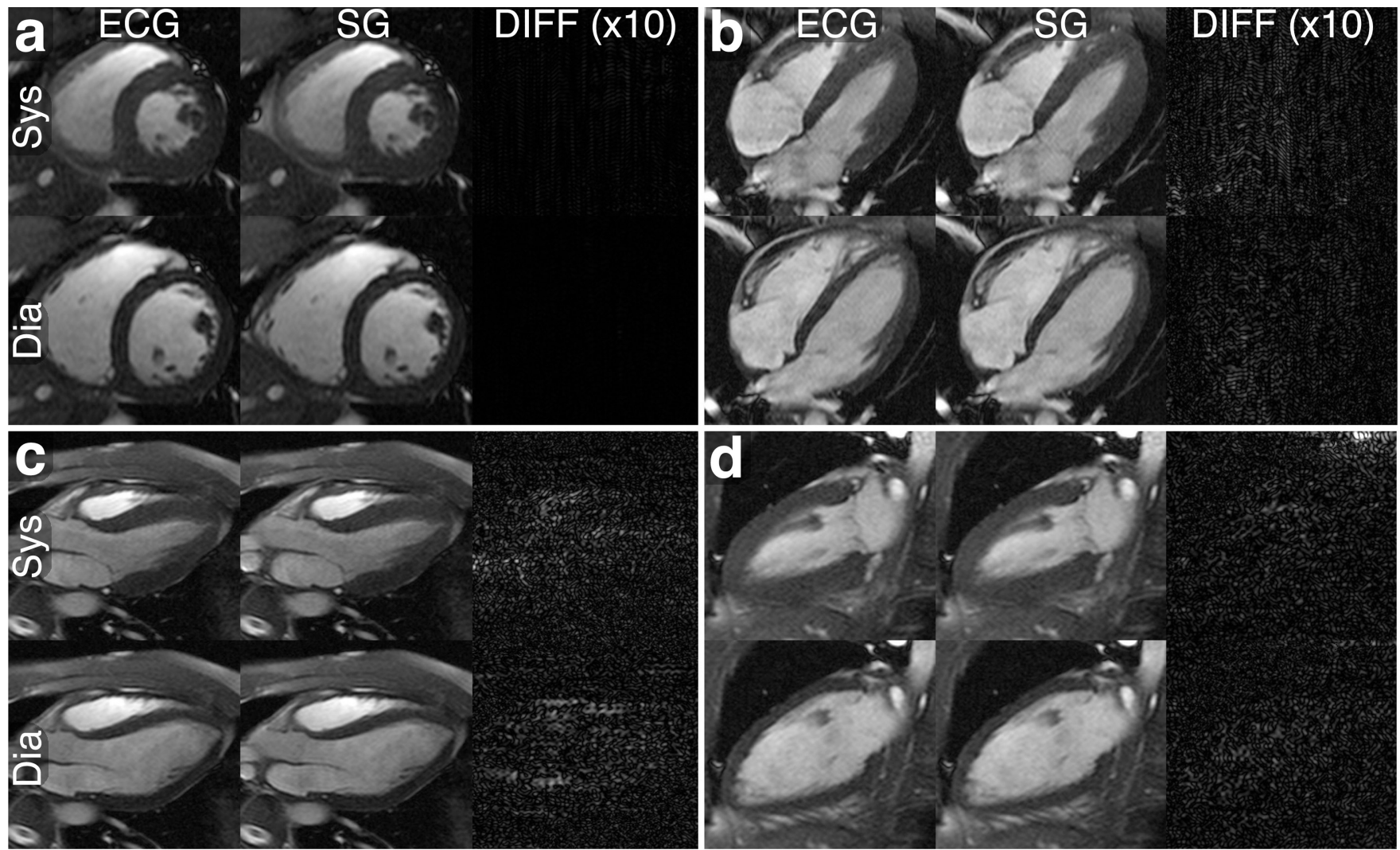

Figure 5.

(a) Short-axis, (b) four-chamber, (c) three-chamber, and (d) two-chamber ATR-SSFP acquisitions from a single volunteer study were reconstructed with ECG gating and self gating (SG). Systolic (Sys) and diastolic (Dia) images are shown for each of the four scan planes. The difference of ECG and self-gating reconstructions is shown for each case, scaled by a factor of 10 . For each scan plane, the same window and level settings were used to display all images. 

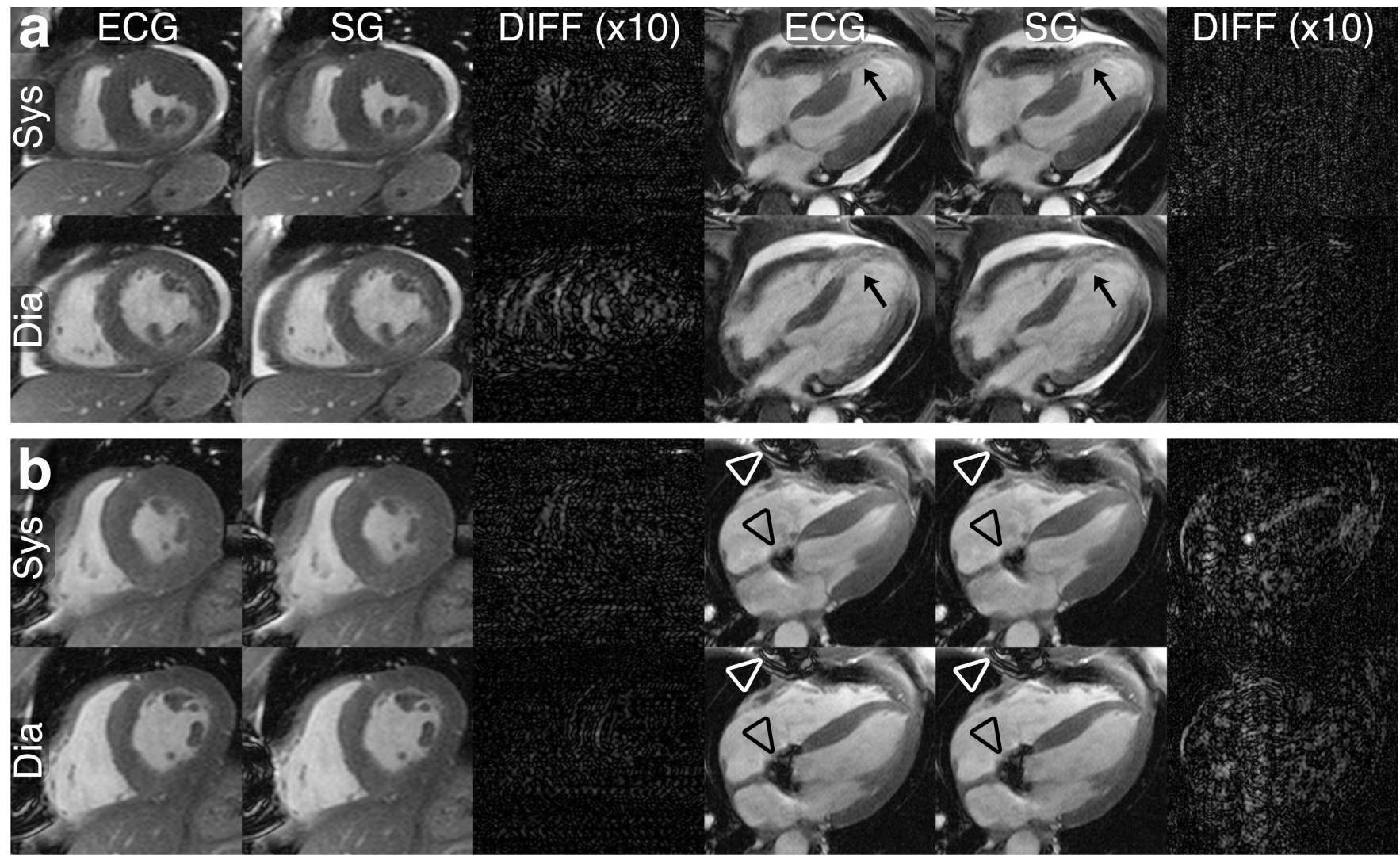

Figure 6.

Short-axis (left) and four-chamber (right) ATR-SSFP images from two different patient studies (a-b) performed after contrast injection. (a) Systolic (Sys) and diastolic (Dia) images of a patient with an apical septal infarct were reconstructed with ECG gating and self gating (SG). The difference of ECG and SG reconstructions is shown for each case, scaled by a factor of 10. Interestingly, a region of hyperintensity in the interventricular septal wall (black arrows) and apex corresponded to regions of scarring found in clinical delayed enhancement scans. (b) Systolic and diastolic images of a patient with aortic valve replacement were reconstructed with ECG gating and self gating. The difference of ECG and SG reconstructions is shown for each case, scaled by a factor of 10. Signal dropouts (arrowheads) are due to the aortic valve replacement. 

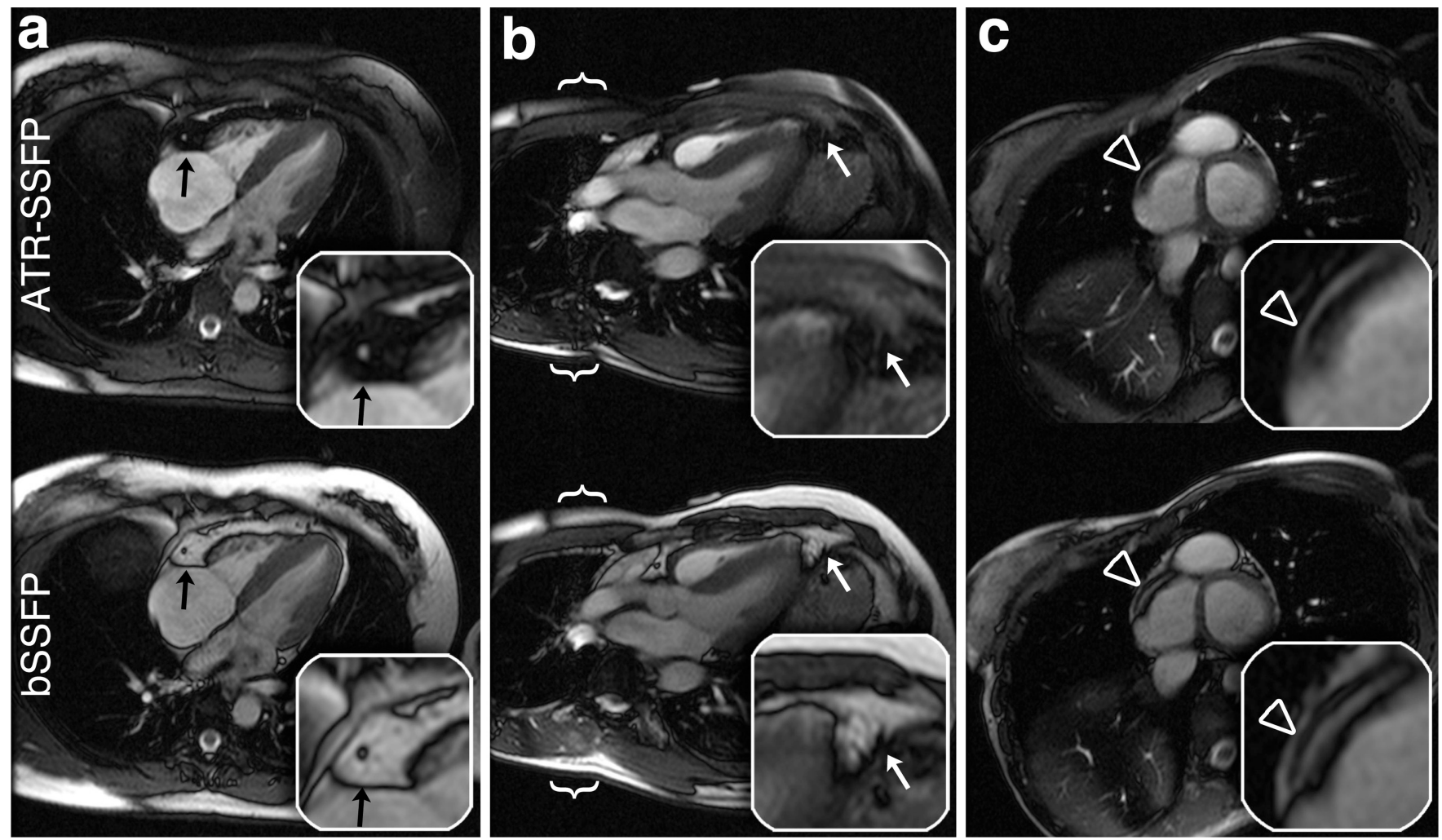

Figure 7.

Self-gated ATR-SSFP images (top) and bSSFP images (bottom) from the same cardiac phase are shown for three different volunteers $(\mathrm{a}-\mathrm{c})$. (a) ATR-SSFP suppresses the signal from epicardial fat surrounding the left and right ventricles, which appears bright in the bSSFP image (black arrows, magnified in inset images). (b) ATR-SSFP suppresses the signal from epicardial fat located at the apex of the heart, which appears bright in the bSSFP image (white arrows, magnified in inset images). Ghosting artifacts near vessels with high flow are slightly worse for ATR-SSFP than bSSFP (bracketed column). (c) A portion of the right coronary artery can be seen in the ATR-SSFP image due to suppression of the signal from surrounding fat (white arrowhead, magnified in inset images). In the bSSFP image, the artery is hypointense due to signal cancellation from the surrounding epicardial fat. 


\section{Table 1}

Reviewer Image Quality Scores and Image Rankings

\begin{tabular}{lcc} 
& Image Quality & Image Rank \\
\hline ECG & $3.29 \pm 0.62$ & $0.06 \pm 0.40$ \\
Self-gating & $3.28 \pm 0.63$ & $-0.06 \pm 0.40$ \\
\hline
\end{tabular}

Quality scores were assigned from 1 (worst) to 4 (best). Ranks were assigned as -1 (worst), 0 (tie), or 1 (best). There were no statistically significant differences $(\mathrm{P}>0.05)$ between ECG and self-gating quality and rank scores. 